\title{
Morphology of intestinal microcirculation in colitis ulcerosa and Crohn's disease
}

\author{
Busikova-Malenovska $\mathrm{P}^{1}$, Labajova $\mathrm{V}^{2}$, Danis $\mathrm{D}^{1}$, Durdik $\mathrm{S}^{3}$, Porubsky $\mathrm{J}^{2}$, Galatova $\mathrm{J}^{4}$ \\ Cythopathos, Institute of Pathological Anatomy, Slovak Health University, Bratislava, Slovakia. \\ bll@fmed.uniba.sk
}

\begin{abstract}
Aim of the work: To describe the state of microcirculation in the intestinal wall in ulcerative colitis and Crohn's disease in own material.

Materials and methods: For morphological examination, we obtained tissue samples from both diseases during bioptic bowel examination from 11 patients. We fixed the samples with $10 \%$ buffered formalin, de-hydrated and covered with paraffin. From paraffin blocks, we made histological sections about $5 \mu \mathrm{m}$ thick with a microtome. They were always stained with haematoxylin and eosin. For immunohistochemistry sections, we either did or did not revitalise the sections according to the manufacturer's recommendations for specific to-reagents. For immunohistochemic examinations, we revitalised the sections by acquiring the heat-induced epitope in DakoCytomation Target Retrieval Solution, Code No S 1700, or in mmol.I-1 citrate buffer, pH 6.0. We did not dry the sections during such procedure. We used antibodies as follows: Monoclonal mouse antibodies against human von Willebrand factor, Clone F8/86 (DakoCytomation, Denmark), Monoclonal mouse antibodies against smooth muscle actine, Clone HHF35 (DakoCytomation, Denmark). We used detection system Dako EnVision ${ }^{T M}$ + Dual Link System-HPR (Dako, Denmark) or Dako Liquid DAB + Substrate Chromogen System (Dako, Denmark) to display areas of specific connection of antibodies. Antibodies against von Willebrand factor react in our samples with the endothelium of vessels and with precursors in the lining of peaks of villi. We have also seen a diffuse positive reaction. Lymphatic vessels do not display monoclonal antibodies (Fig. 4, Ref. 8). Full Text in PDF www.elis.sk. Key words: intestinal microcirculation, colitis ulcerosa, Crohn's disease.
\end{abstract}

In inflammatory bowel diseases, the anatomical and functional relations of different barriers have a striking relationship to microcirculation of the intestinal wall. Colitis ulcerosa and Crohn's disease are amongst the chronic inflammatory bowel diseases. These represent a pressing problem for global healthcare. At the beginning of 2011, the database of the U.S. National Library of Medicine National, Institutes of Health Pubmed.gov, had over 8,000 information sources on this issue. About $25 \%$ thereof deal with the morphological picture of both diseases. Almost three-quarters of the total of such information sources describe their pathogenesis. To a large extent, the essence of both diseases remains unclear. Intercellular relationships and associated tight junction (1) generally draw much attention. The second point of interest is the state of blood circulation in the affected mucosa. Surprisingly, in the PubMed. gov database, only 31 information sources relate to this problem.

\section{Aim of the work}

To describe the state of microcirculation in the intestinal wall in ulcerative colitis and Crohn's disease in own material.

${ }^{1}$ Cythopathos, Institute of Pathological Anatomy, Slovak Health University, Bratislava, ${ }^{2}$ Institute of Pathological Anatomy, Faculty of Medicine, Comenius University, Bratislava, ${ }^{3} \mathrm{Clinic}$ of Oncological Surgery, Bratislava, and ${ }^{4}$ Institute of Foreign Language, Faculty of Medicine, Comenius University, Bratislava, Slovakia

Address for correspondence: P. Malenovska-Busikova, MD, Hviezdoslavova 31, SK-901 01 Malacky, Slovakia. Phone: +421.918402476

\section{Materials and methods}

For morphological examination of 11 patients, we obtained tissue samples from both diseases via bioptic bowel examination. We fixed the samples with $10 \%$ buffered formalin, de-hydrated them and covered with paraffin. From paraffin blocks, we made histological sections about $5 \mu \mathrm{m}$ thick with a microtome. They were always stained with haematoxylin and eosin. For immunohistochemistry sections, we either did or did not revitalise the sections according to the manufacturer's recommendations for specific to-reagents. For immunohistochemic examinations, we revitalised the sections by acquiring the heat-induced epitope in DakoCytomation Target Retrieval Solution, Code No S 1700, or in mmol.1-1 citrate buffer, $\mathrm{pH}$ 6.0. We did not dry the sections during such procedure.

We used antibodies as follows:

- Monoclonal mouse antibodies against human von Willebrand factor, Clone F8/86 (DakoCytomation, Denmark),

- Monoclonal mouse antibodies against smooth muscle actin, Clone HHF35 (DakoCytomation, Denmark).

We used detection system Dako EnVision ${ }^{\mathrm{TM}}+$ Dual Link System-HPR (Dako, Denmark) or Dako Liquid DAB + Substrate Chromogen System (Dako, Denmark) to display areas of specific connection of antibodies. 


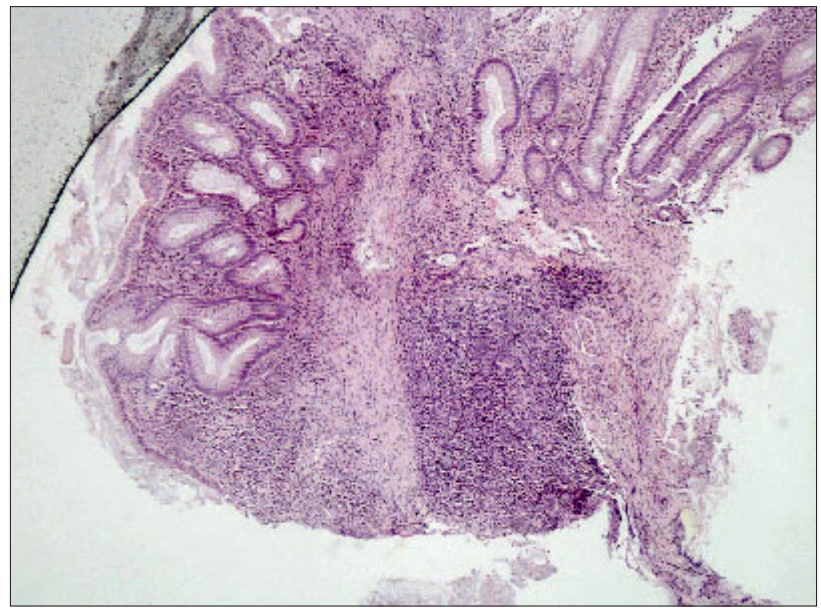

Fig. 1. Transparent picture of the entire bioptic sample of a tissue from a patient with colitis ulcerosa. As well as a layer of glands, fibrosis of lamina propria mucosae and a focus of lymphoid tissue (HE, $x$ 40).

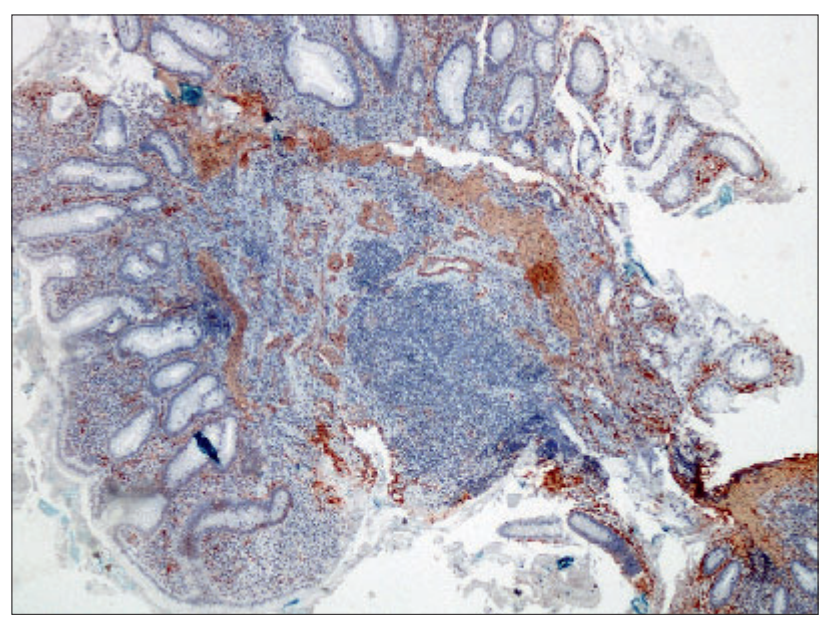

Fig. 2. Parallel section as in Fig 1. Positive reaction in walls of vessels, and also round cells around them. Antibodies against (F-VIII. x. 40).

\section{Results}

Bioptic samples of tissue do not contain deeper parts of intestinal wall; however, sometimes they also take a part of submucosis under lamina muscularis mucosae. In the morphologic image of chronic inflammatory bowel diseases, regardless of whether the samples were obtained from colitis ulcerosa or from Crohn's disease, the manifestations of intestinal microcirculation activity are striking. In Crohn's disease, as well as in cases of focuses of chronic inflammatory infiltrate, there are variously large focuses of fibrosis in lamina propria mucosae.

In haematoxylin and eosin stained sections, regardless of whether the samples were acquired from colitis ulcerosa or Crohn's disease, there is an abundant polymorfonuclear infiltrate in lamina propria mucosae, sometimes also in abscesses in crypts. In addition to inflammatory infiltrate, there are also fibrocytes and fibroblasts (Fig. 1). Submucosis is affected by oedema, fibrosis and vasodilation. When applying antibodies against von Willebrand factor,

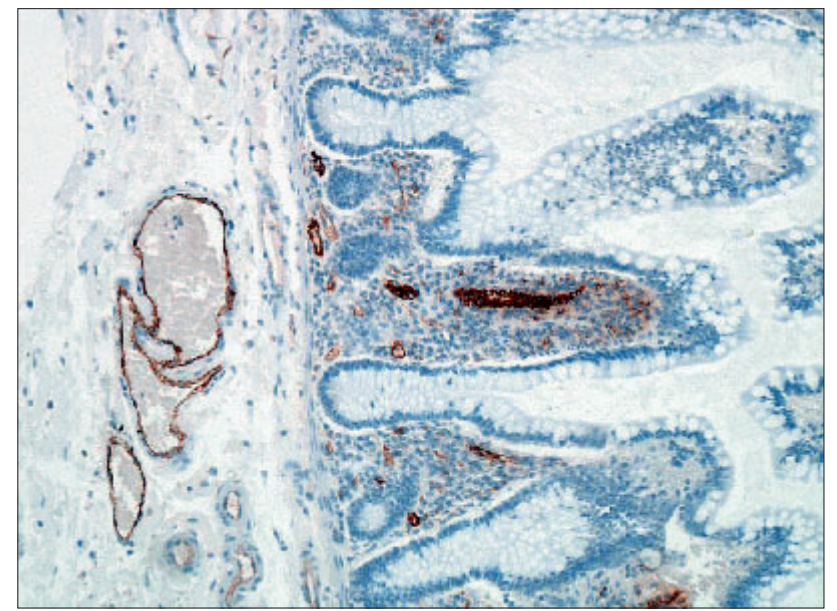

Fig. 3. Antibodies against von Willebrand factor react very intensely with endothelium of microcirculation of lamina propria mucosae as well as with proliferating endothelium of veins in the submucosis. Specifically, the undifferentiated cells react positively in the stroma in the peaks of villi. In small arteries in the submucosis, there is a weak positive reaction. In lymphatic vessels, the reaction is negative. Anti (FVIII. x 200).

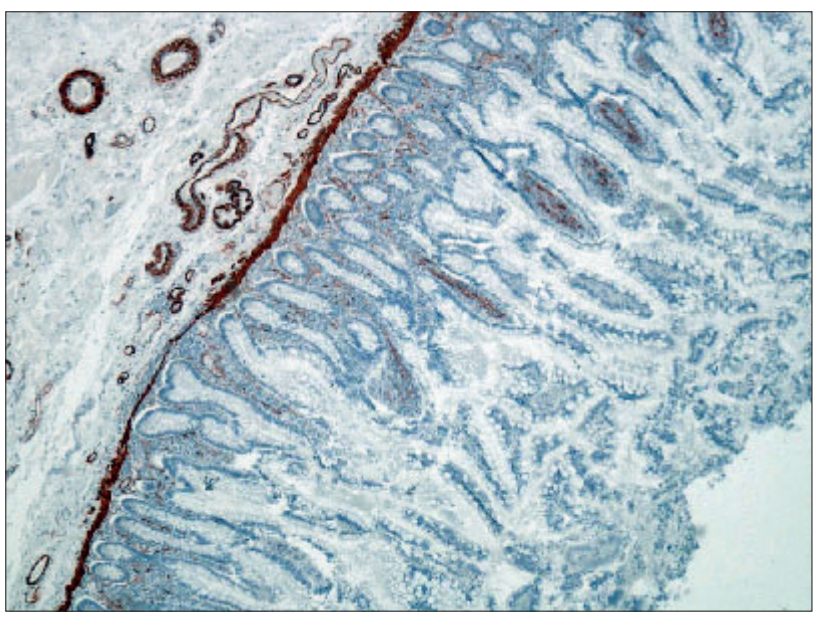

Fig. 4. Antibodies against SMA (smooth muscle actin) react with lamina muscularis mucosae, with the walls of small vessels in the submucosis, also with the vessels in the stroma of villi. Anti SMA. 40x.

the microscopic image of colitis ulcerosa shows the positive reaction of walls of lumen surface of vascular structures. In addition to the displayed postcapilar venules, veins and capillaries, in the apex of villi, we can see positively reacting mononuclear cells without lumen.

Immunohistochemical examination with antibodies against smooth muscle actin cells in human samples with colitis ulcerosa, reveals a positive reaction around glands and crypts mainly around the ulcers. On the edge of lymphoid tissue, the stroma shows a reaction with the walls of vascular structures. In Crohn's disease, the positive finding is found in lamina muscularis mucosae, where we distinguish both longitudinal and transversely oriented structures. Sometimes, bundles of smooth muscle fibres are emitted into lamina propria mucosae. In lamina muscularis mucosae, we 
can also see crevicular spaces with negative reaction. They include tiny blood vessels, the walls of which react with antibodies against actin. In the inter-crypt area, we can see fibrous material of varying thickness.

People with colitis ulcerosa are affected mainly by fibrosis of lamina propria mucosae whereas the focuses of lymphoid tissue originate rarely (Fig. 2) while being more frequent in Crohn's disease. In case of both diseases, in lamina propria mucosae as well as around the affected areas, there is a variously thick mononuclear infiltrate. When using antibodies against F VIII, the endothelium of blood vessels of different calibre responds positively, while this reaction is not seen in the endothelium of lymphatic vessels (Figs 3 and 4). Antibodies against von Willebrand antigen (F-VIII) react very intensely with the endothelium of microcirculation of lamina propria mucosae, as well as with the proliferating endothelium of vein in the submucosis. In the stroma, particularly in the peaks of villi, the specifically undifferentiated cells react positively. In small arteries within the submucosis, there is a weak positive reaction. In lymphatic vessels, the reaction is negative.

Antibodies against actin within smooth muscle react in lamina muscularis mucosae with walls of small vessels in the submucosis, and with blood vessels in the stroma of the villi.

\section{Discussion}

Antibodies against von Willebrandov factor react with endothelium, megakaryocytes and megakaryoblasts, as well as with subendotelium matrix of vessels walls. The VIII factor enables the adherence of platelets to the damaged endothelium (3). In our samples, these antibodies react with the endothelium of vessels and with its not yet undifferentiated precursors in the lining of peaks of villi. The presence of such cells could possibly have a connection with angiogenesis, which is increased in inflammatory bowel diseases (4). In this area, we can also see a diffusely positive reaction which can specifically show platelets and thus contribute to the development of the condition of hypercoagulation in patients with IBD (5).
Antibodies against actin within smooth muscle cells react with lamina muscularis mucosae and smooth muscles of vessels. In our samples, we can also find a positive, focus-interrupted structure in the lining of apices of villi. Nevertheless it is questionable whether this relates to intestinal microcirculation (either blood or lymphatic) or to independent muscle fibres (6).

Lymphatic vessels do not display monoclonal antibodies. A solution for their display can lie in the use of antibodies against podoplanine (D2-40) which specifically prove only their endothelium $(7,8)$. This would contribute to a better understanding of blood and lymphatic microcirculation in the pathogenesis of IBD, particularly in colitis ulcerosa.

\section{References}

1. Edelblum KL, Turner JR. The tight junction in inflammatory disease: communication breakdown. Curr Opin Pharmacol 2009; 9 (6): 715-720.

2. Rubin E, Farber JL. Pathology. J.B. Lippincott Company, 1988; 692-700.

3. Wang JW, Eikenboom J. Von Willebrand disease and Weibel-Palade bodies. Hamostaseologie 2010; 30 (3): 150-155.

4. Danese S, Sans M, de la Motte C, Graziani C, West G, Phillips MH, Pola R, Rutella S, Willis J, Gasbarrini A, Fiocchi C. Angiogenesis as a novel component of inflammatory bowel disease pathogenesis. Gastroenterology 2006; 130 (7): 2060-2073.

5. Talbot RW, Heppell J, Dozois RR, Beart RW Jr. Vascular complications of inflammatory bowel disease. Mayo Clin Proc 1986; 61 (2): $140-145$.

6. Kaiserling E, Kröber S, Geleff S. Lymphatic vessels in the colonic mucosa in ulcerative colitis. Lymphology 2003; 36 (2): 52-61.

7. Schacht V, Dadras SS, Johnson LA, Jackson DG, Hong YK, Detmar M. Up-regulation of the lymphatic marker podoplanin, a mucin-type transmembrane glycoprotein, in human squamous cell carcinomas and germ cell tumors. Am J Pathol 2005; 166 (3): 913-921.

8. Linares P, Gisbert J. Role of Growth Factors in the Development of Lymphangiogenesis Driven by Inflammatory Bowel Disease. Inflamm Bowel Dis 2010. 